Research Article

\title{
Factors Related to Superior and Inferior Hemifield Defects in Primary Open-Angle Glaucoma
}

\author{
Remi Takeuchi (D), Nobuko Enomoto (D), Kyoko Ishida (D), Ayako Anraku (D), \\ and Goji Tomita (iD \\ Department of Ophthalmology, Toho University Ohashi Medical Center, 2-22-36 Ohashi, Meguro-ku, Tokyo 153-8515, Japan \\ Correspondence should be addressed to Nobuko Enomoto; nobuko.enomoto@med.toho-u.ac.jp
}

Received 4 October 2018; Revised 9 March 2019; Accepted 19 March 2019; Published 14 April 2019

Academic Editor: Tomasz Zarnowski

Copyright (c) 2019 Remi Takeuchi et al. This is an open access article distributed under the Creative Commons Attribution License, which permits unrestricted use, distribution, and reproduction in any medium, provided the original work is properly cited.

\begin{abstract}
Purpose. We aimed to investigate factors related to superior and inferior hemifield defects in primary open-angle glaucoma (POAG). Methods. Sixty-seven subjects with newly diagnosed, untreated POAG underwent optical coherence tomography (OCT) of the disc area, macular ganglion cell complex (mGCC), and circumpapillary retinal nerve fiber layer (cpRNFL) thickness within 6 months of the visual field (VF) test. Based on the VF and OCT results, 40 subjects had a superior and 27 an inferior hemifield defect. Clinical data including visual acuity, refractive error, disc hemorrhage, VF indexes, and medical history were recorded. Results. Average mGCC thickness corresponding to the defective hemifields was thinner in the superior VF defect group than in the inferior VF defect group $(P=0.003)$. Average total deviation (TD) was comparable between the two groups. However, the superior VF defect group had a higher prevalence of defects $(P=0.001)$ and lower TD $(P=0.002)$ within central 5 degrees of VF than the inferior VF defect group. In multivariate regression analyses, the temporal-lower and inferior-temporal cpRNFL thicknesses were significant contributing factors to the inferior mGCC thickness in the superior VF defect group. In the inferior VF defect group, the disc area, family history of glaucoma, and temporal-upper cpRNFL thickness contributed to the superior mGCC thickness. Conclusion. The inferior mGCC thickness corresponding to the superior hemifield defect group was significantly thinner than the superior mGCC thickness corresponding to the inferior hemifield defect group. The factors related to the reduction of the corresponding mGCC thickness may differ between superior VF defect and inferior VF defect groups.
\end{abstract}

\section{Introduction}

Glaucoma is characterized by chronic progressive optic neuropathy with corresponding and characteristic patterns of visual field (VF) defects. Mikelberg and Drance found that $70 \%$ glaucomatous eyes had initial damage limited to a single hemifield and $57 \%$ still had only a single hemifield defect at the completion of follow-up [1]. Previous studies have reported a 6:1 prevalence of superior over inferior paracentral VF defects and similar or more inferior VF defects in conventional peripheral VF defects $[2,3]$. Localized optic disc change associated with glaucomatous paracentral scotomas lies closer to the papillomacular bundle than that associated with peripheral VF loss [4-8]. This may be explained by the characteristic distribution of retinal fiber layer axons in the retina and asymmetric VF defects, which often occur between the superior and inferior hemifields.
Paracentral defects have been reported to occur more commonly in eyes with intraocular pressure (IOP) within the statistically normal range (normal-pressure glaucoma) than in those with high-pressure glaucoma $[9,10]$, although other reports have been nonconfirmatory [11, 12]. Thus, many studies have compared structural and clinical differences in paracentral and peripheral scotoma [1-3, 13-17]. However, few studies have investigated structural and clinical factors contributing to the differences between glaucomatous eyes with superior hemifield defects and inferior hemifield defects. Moreover, the definition of hemifield defects in previous studies [2, 3, 13-17] was based on VF results only. However, even in apparently normal hemifield, structural damages were detected by image analysis tools, such as optical coherence tomography (OCT) $[18,19]$. The purpose of this study was to investigate the factors related to superior and inferior hemifield defects 
defined based on VF and OCT results in patients with primary open-angle glaucoma (POAG) or normal tension glaucoma (NTG).

\section{Materials and Methods}

2.1. Study Subjects. This study was approved by the Ethics Committee of Toho University Ohashi Medical Center (number H17036), and all study conducts adhered to the tenets of the Declaration of Helsinki. We retrospectively reviewed the medical records of patients with glaucoma from the Department of Ophthalmology Outpatient Clinic at Toho University Ohashi Medical Center (Tokyo, Japan) between July 2007 and August 2017. The inclusion criteria were (1) clinical diagnosis of untreated POAG or NTG with a hemifield defect, (2) OCT measurements such as macular ganglion cell complex (mGCC) and circumpapillary retinal nerve fiber layer (cpRNFL) thicknesses corresponding to defective hemifields showing significant $(P<0.05)$ abnormality, and both mGCC thickness and cpRNFL thickness corresponding to normal hemifields within the normal limit, (3) a best-corrected visual acuity of at least 20/25, and (4) a spherical refractive error between -6.00 and +3.00 diopters (D), a refractive cylindrical error within $2.00 \mathrm{D}$. The exclusion criteria were (1) history of intraocular surgery and (2) presence of intraocular diseases other than POAG or NTG, or other diseases affecting the VF (e.g., pituitary lesions, demyelinating disease, or diabetic retinopathy). If both eyes of a patient satisfied the inclusion criteria, the right eye was selected.

All patients underwent OCT measurements of both mGCC and cpRNFL thicknesses within 6 months of the Humphrey field analyzer (HFA; Carl Zeiss Meditec Inc., Dublin, CA, USA) measurements. In all patients, we recorded age, sex, visual acuity, spherical equivalent refractive error, untreated IOP, central corneal thickness (CCT), OCT disc area, disc hemorrhage (DH) during followup, family history of glaucoma, history of systemic hypertension and diabetes mellitus, and mean deviation (MD), and pattern standard deviation (PSD) in the standard automated perimetry with the HFA. The IOP was measured with a Goldmann applanation tonometer, and the mean untreated IOP was calculated by three measurement values obtained on 3 separate days. If the IOP measurement exceeded $21 \mathrm{mmHg}$ even once, we diagnosed the patient with POAG [20].

\subsection{Ganglion Cell Complex and Retinal Nerve Fiber Layer} Thickness Measurements. All OCT measurements were performed with the RTVue-100 Spectral-domain OCT (software version 4.0, Optovue, Inc., Fremont, CA, USA), which uses a scanning laser diode to emit a scan beam with a wavelength of $840 \pm 10 \mathrm{~nm}$. This system provides images of ocular microstructures.

In this study, the GCC scanning protocol was used to measure mGCC thickness. The GCC protocol consists of one horizontal and 15 vertical line scans that cover a $7 \times 7 \mathrm{~mm}$ region. Each GCC scan captures 15,000 data points within
0.6 seconds, and a $6 \times 6 \mathrm{~mm}$ map (corresponding to approximately $20^{\circ}$ on the visual field map) is created. The mGCC thickness was measured from the internal limiting membrane to the outer inner plexiform layer boundary, and the OCT system provided overall superior and inferior hemifield averages.

The optic nerve head $(\mathrm{ONH})$ protocol was used for cpRNFL thickness measurements. Using the fundus picture generated by OCT (a video baseline protocol), we manually traced ONH contours. The RNFL thickness was automatically measured along a $3.45 \mathrm{~mm}$-diameter circle centered at the center of the optic disc. A total of 775 A-scans was obtained along this circle. We obtained the average thickness of cpRNFL in the superior and inferior hemifields, and the superior-temporal (ST), temporal-upper (TU), temporallower (TL), and inferior-temporal (IT) average thicknesses of cpRNFL, which were measured automatically by OCT. In addition, the disc area was obtained from disc parameters (Figure 1).

A trained operator obtained good quality OCT images from each subject after pupillary dilation. Images were excluded from analyses when the signal strength index was low $(<40)$, when segmentation errors occurred, or when the scan circle was not centered at the optic disc.

2.3. Definition of VF Defects. Standard automated perimetry was performed with the HFA using the 30-2 Swedish Interactive Threshold Algorithm. VF tests were considered reliable when fixation losses were $<20 \%$, false positives were $<15 \%$, and false negatives were $<25 \%$. A glaucomatous functional hemifield defect was defined by the presence of three or more significant $(P<0.05)$, nonedge-contiguous points, with at least one highly significant $(P=0.01)$ point in the pattern deviation plot, along with grading outside the normal limits in the glaucoma hemifield test [21]. A normal hemifield was defined as two or less significant $(P<0.05)$, nonedge-contiguous points in the pattern deviation plot $[22,23]$.

2.4. Evaluation of Central VF Defect. In the pattern deviation plot of the HFA, we examined for the presence of significant $(P<0.05)$ points in 16 points within central 10 degrees and 4 points within central 5 degrees (Figure 1). Average total deviation (TD) values of superior (C8-sup) and inferior 8 points (C8-inf) within central 10 degrees and superior (C2sup) and inferior 2 points (C2-inf) within central 5 degrees were calculated (Figure 1).

2.5. Statistical Analyses. Data are reported as mean\pm standard deviation. The normality of the data was examined using the Shapiro-Wilk test, and nonparametric tests were performed for nonnormally distributed data. Average TD values for the superior or inferior hemifield were calculated. Age, spherical equivalent refractive error, untreated IOP, CCT, disc area, MD, PSD, averages of superior mGCC and cpRNFL thicknesses corresponding to the inferior VF defect, averages of inferior $\mathrm{mGCC}$ and cpRNFL 

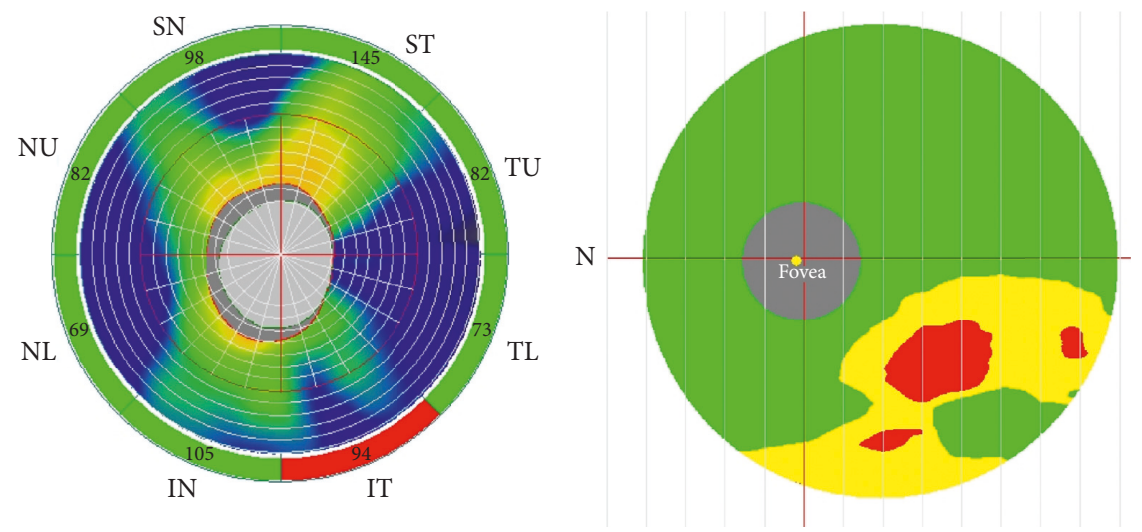

(a)
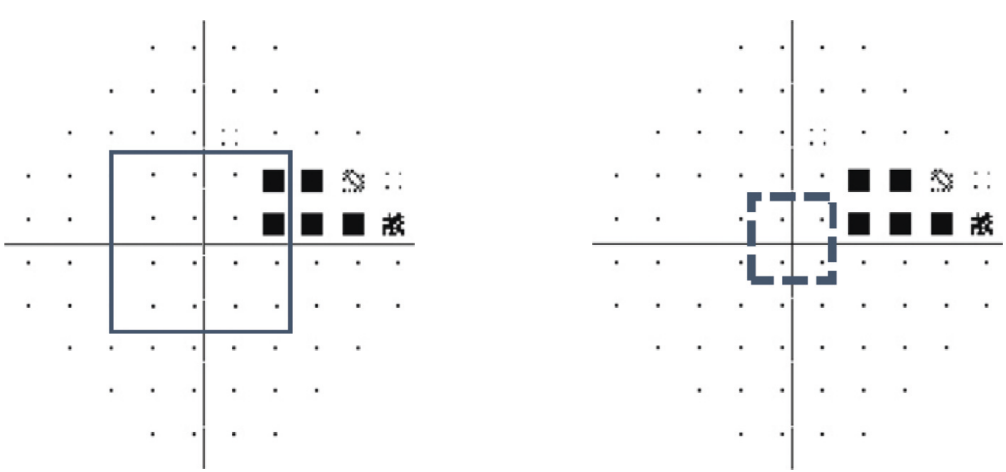

(b)

Figure 1: (a) A representative eye with glaucoma with superior hemifield defects measured by spectral-domain optical coherence tomography (SDOCT). Circumpapillary retinal nerve fiber layer (cpRNFL) thickness map (left) and macular ganglion cell complex (mGCC) thickness map (right). ST: superior-temporal; TU: temporal-upper; TL: temporal-lower; IT: inferior-temporal; IN: inferior-nasal; NL: nasallower; NU: nasal-upper; SN: superior-nasal. (b) Evaluation of the central visual field defect. The VF defects correspond to the cpRNFL and mGCC maps at the top panels. In the pattern deviation plot of the visual field measured by the Humphrey field analyzer, the prevalence of significant $(P<0.05)$ points in 16 points within central 10 degrees and 4 points within central 5 degrees is measured. (Left) The central visual field defect is defined because of at least 1 significant point lying in 16 points (squeal line) within central 10 degrees. (Right) The central visual field defect is not defined because of no significant point lying in 4 points (squeal dot line) within central 5 degrees.

thicknesses corresponding to the superior VF defect, average TD values for the superior and inferior hemifields, C8-sup and -inf, and C2-sup and -inf corresponding to the superior or inferior hemifield defect between the two groups were compared using the Mann-Whitney $U$-test. Sex ratio, family history of glaucoma, DH, systemic factors, presence of significant $(P<0.05)$ points in the pattern deviation plot in 16 points within central 10 degrees (defects within 10 degrees), and presence of significant $(P<0.05)$ points in the pattern deviation plot in 4 points within central 5 degrees (defects within 5 degrees) between the two groups were compared using the chi-square test or Fisher's exact test. Univariate and multivariate regression analyses were used to determine factors contributing to mGCC thickness in each VF defect group. Explanatory variables were age, sex, visual acuity, spherical equivalent refractive error, untreated IOP, CCT, disc area, DH, family history of glaucoma, history of systemic hypertension and diabetes mellitus, ST, TU, TL, and IT RNFL thickness, and C2-sup and -inf. The factors that showed significant probability lower than 0.2 were included in multiple stepwise regression analysis as explanatory variables. Statistical significance was accepted at $P<0.05$. All analyses were performed using statistical software (SPSS version 19.0 for Windows; SPSS Inc., Chicago, IL).

\section{Results}

From the chart review, 106 patients who had hemifield defect consistent with the VF criteria were located. Among them, 67 eyes of 67 patients met the hemisphere disorder criteria of OCT corresponding to the VF defect.

The subjects' characteristics are presented in Table 1. Forty of 67 subjects (60\%) had a superior VF defect, and 27 $(40 \%)$ had an inferior VF defect. There was no significant difference in age, sex, spherical equivalent refractive error, untreated IOP, CCT, MD, PSD, disc area, DH, family history of glaucoma, and history of hypertension and diabetes mellitus between the groups (Table 2). However, the average of mGCC thickness corresponding to the defective hemifields was significantly thinner in the superior VF defect group than in the inferior VF defect group $(P=0.003)$ (Table 2).

There was no significant difference in the prevalence of "defects within 10 degrees" between the two groups. However, the superior VF defect group had a higher 
TABle 1: Patient characteristics.

Demographic factors

Age (years)

$56.15 \pm 11.73$

Sex: male/female

$24(35.8 \%) / 43(64.2 \%)$

Family history of glaucoma: yes/no

$14(20.9 \%) / 53(79.1 \%)$

Ocular factors

POAG/NTG

Visual acuity

Spherical equivalent (D)

Untreated IOP (mmHg)

CCT $(\mu \mathrm{m})$

$\mathrm{DH}$ : presence/absence

Disc area $\left(\mathrm{mm}^{2}\right)$

cpRNFL thickness corresponding to defective

hemifield $(\mu \mathrm{m})$

mGCC thickness corresponding to defective

hemifield $(\mu \mathrm{m})$

$5(7.5 \%) / 62(92.5 \%)$

$1.19 \pm 0.05$

$-2.65 \pm 2.53$

$15.20 \pm 2.26$

$526.28 \pm 33.98$

$3(4.5 \%) / 64(95.5 \%)$

$2.04 \pm 0.44$

$77.10 \pm 10.06$

$78.04 \pm 8.67$

Perimetric parameters

$\mathrm{MD}(\mathrm{dB})$

PSD (dB)

Average TD values corresponding to defective hemifield $(\mathrm{dB})$

Prevalence of defects within 10 degrees: yes/no

Prevalence of defects within 5 degrees: yes/no

Average TD values of superior within 10 degrees (C8-

sup) (dB)

Average TD values of inferior within 10 degrees (C8-

inf) (dB)

Average TD values of superior within 5 degrees (C2-

sup) (dB)

Average TD values of inferior within 5 degrees (C2inf) (dB)

Systemic factors

Hypertension: yes/no

Diabetes mellitus: yes/no

$15(22.4 \%) / 52(77.6 \%)$

$7(10.4 \%) / 60(89.6 \%)$

Continuous variables are expressed as $N$ (percentage), mean \pm SD. POAG: primary open-angle glaucoma, NTG: normal tension glaucoma, IOP: intraocular pressure, CCT: central corneal thickness, DH: disc hemorrhage, cpRNFL: circumpapillary retinal nerve fiber layer, mGCC: macular ganglion cell complex, MD: mean deviation, PSD: pattern standard deviation, and TD: total deviation.

prevalence of "defects within 5 degrees" $(P=0.001)$ than the inferior VF defect group (Table 2).

The average TD of the superior and inferior VF defect was similar between the groups. There was no significant difference between the C8-sup corresponding to the defective hemifields of the superior VF defect group and the C8-inf corresponding to the defective hemifields of the inferior VF defect group. However, the C2-sup corresponding to the defective hemifields of the superior VF defect group was significantly lower than $\mathrm{C} 2$-inf corresponding to the defective hemifields of the inferior VF defect group $(P=0.002)$ (Table 2).

For the inferior mGCC thickness in the superior VF defect group, ST, TU, TL and IT RNFL thickness, and C2sup were selected as significant related factors by univariate regression analysis. In multivariate analysis, ST, TU, TL and IT RNFL thickness, and C2-sup were included as explanatory variables; the TL RNFL thickness (slope $=0.47 \mu \mathrm{m} / \mu \mathrm{m}$, standard partial regression coefficient $(\beta)=0.55$, 95\% confidence interval $(\mathrm{CI})=0.25$ to 0.70 , and $P<0.001)$ and the IT RNFL thickness (slope $=0.16 \mu \mathrm{m} / \mu \mathrm{m}, \beta=0.27,95 \% \mathrm{CI}=0.01$ to 0.31 , and $P=0.035$ ) were selected as significant contributing factors to the inferior mGCC thickness in the superior VF defect group (Table 3). On the other hand, for the superior mGCC thickness in the inferior VF defect group, family history of glaucoma, spherical equivalent refractive error, disc area, ST and TU RNFL thickness, and C2-sup were selected as significant related factors by univariate regression analysis. In multivariate analysis, family history of glaucoma, spherical equivalent refractive error, disc area, ST and TU RNFL thickness, and C2-sup were included as explanatory variables; family history of glaucoma (slope $=5.93 / \mu \mathrm{m}, \beta=0.38,95 \% \mathrm{CI}=2.00$ to 9.85 , and $P=0.005)$, the disc area (slope $=5.15 \mu \mathrm{m} / \mathrm{mm}^{2}, \beta=0.35$, $95 \% \mathrm{CI}=1.43$ to 8.87 , and $\beta=0.009$ ), and TU RNFL thickness (slope $=0.41 \mu \mathrm{m} / \mu \mathrm{m}, \mu=0.64,95 \% \mathrm{CI}=0.25$ to 0.56 , and $P<0.001)$ were selected as significant contributing factors to the superior mGCC thickness in the inferior VF defect group (Table 4).

\section{Discussion}

In this study, we investigated factors related to superior and inferior hemifield defects in patients with POAG or NTG. 
TABLE 2: Comparison of clinical characteristics between the superior hemifield and inferior hemifield defect groups.

\begin{tabular}{|c|c|c|c|}
\hline & Superior visual field defect group & Inferior visual field defect group & $P$ value \\
\hline \multicolumn{4}{|l|}{ Demographic factors } \\
\hline Age (years) & $54.33 \pm 13.31$ & $58.85 \pm 8.39$ & 0.205 \\
\hline Sex: male/female & $15(37.5 \%) / 25(62.5 \%)$ & $9(33.3 \%) / 18(66.7 \%)$ & 0.727 \\
\hline Family history of glaucoma: yes/no & $9(22.5 \%) / 31(77.5 \%)$ & $5(18.5 \%) / 22(81.5 \%)$ & 0.694 \\
\hline \multicolumn{4}{|l|}{ Ocular factors } \\
\hline Visual acuity & $1.18 \pm 0.06$ & $1.19 \pm 0.04$ & 0.340 \\
\hline Spherical equivalent (D) & $-2.50 \pm 2.78$ & $-2.87 \pm 2.15$ & 0.711 \\
\hline Untreated IOP (mmHg) & $15.57 \pm 2.40$ & $14.66 \pm 1.97$ & 0.107 \\
\hline $\mathrm{CCT}(\mu \mathrm{m})$ & $524.13 \pm 33.75$ & $529.48 \pm 34.70$ & 0.385 \\
\hline DH: presence/absence & $1(7.5 \%) / 39(92.5 \%)$ & $2(7.4 \%) / 25(92.6 \%)$ & 0.354 \\
\hline Disc area $\left(\mathrm{mm}^{2}\right)$ & $2.07 \pm 0.45$ & $2.00 \pm 0.42$ & 0.544 \\
\hline $\begin{array}{l}\text { cpRNFL thickness corresponding to the defective } \\
\text { hemifield }(\mu \mathrm{m})\end{array}$ & $75.27 \pm 8.02$ & $79.83 \pm 12.14$ & 0.050 \\
\hline $\begin{array}{l}\text { mGCC thickness corresponding to the defective } \\
\text { hemifield }(\mu \mathrm{m})\end{array}$ & $75.86 \pm 9.44$ & $81.27 \pm 6.25$ & 0.003 \\
\hline \multicolumn{4}{|l|}{ Perimetric parameters } \\
\hline $\mathrm{MD}(\mathrm{dB})$ & $-3.30 \pm 3.72$ & $-2.07 \pm 1.73$ & 0.609 \\
\hline PSD (dB) & $6.94 \pm 4.81$ & $5.90 \pm 3.24$ & 0.596 \\
\hline $\begin{array}{l}\text { Average TD values corresponding to defective } \\
\text { hemifield }(\mathrm{dB})\end{array}$ & $-6.51 \pm 7.34$ & $-3.53 \pm 2.53$ & 0.371 \\
\hline Prevalence of defects within 10 degrees: yes/no & $39(97.5 \%) / 1(2.5 \%)$ & $23(85.2 \%) / 4(14.8 \%)$ & 0.081 \\
\hline Prevalence of defects within 5 degrees: yes/no & $22(55.0 \%) / 18(45.0 \%)$ & $4(14.8 \%) / 23(85.2 \%)$ & 0.001 \\
\hline $\begin{array}{l}\text { Average TD values within } 10 \text { degrees corresponding } \\
\text { to defective hemifield }(\mathrm{C} 8)(\mathrm{dB})\end{array}$ & $-8.02 \pm 8.55$ & $-3.93 \pm 4.99$ & 0.066 \\
\hline $\begin{array}{l}\text { Average TD values within } 5 \text { degrees corresponding to } \\
\text { defective hemifield (C2) (dB) }\end{array}$ & $-6.09 \pm 9.37$ & $-1.00 \pm 5.16$ & 0.002 \\
\hline \multicolumn{4}{|l|}{ Systemic factors } \\
\hline Hypertension: yes/no & $8(20.0 \%) / 32(80.0 \%)$ & $7(25.9 \%) / 20(74.1 \%)$ & 0.568 \\
\hline Diabetes mellitus: yes/no & $4(10.0 \%) / 36(90.0 \%)$ & $3(11.1 \%) / 24(88.9 \%)$ & 0.594 \\
\hline
\end{tabular}

Continuous variables are expressed as $N$ (percentage), mean $\pm \mathrm{SD}$, or percentage. * Statistically significant differences between the superior hemifield defect group and inferior hemifield defect group $(P<0.05)$ by the Mann-Whitney $U$-test for continuous variables or Fisher's exact test for categorical data are indicated in bold. IOP: intraocular pressure, CCT: central corneal thickness, DH: disc hemorrhage, cpRNFL: circumpapillary retinal nerve fiber layer, mGCC: macular ganglion cell complex, MD: mean deviation, PSD: pattern standard deviation, and TD: total deviation.

TABle 3: Univariate and multivariate regression analyses for inferior mGCC thickness in the superior visual field defect group.

\begin{tabular}{|c|c|c|c|c|c|c|}
\hline & \multicolumn{2}{|c|}{$\begin{array}{l}\text { Univariate regression } \\
\text { analysis }\end{array}$} & \multicolumn{4}{|c|}{ Multivariate regression analysis } \\
\hline & $r$ & $P$ value & Slope & $\beta$ & $95 \% \mathrm{CI}$ & $P$ value \\
\hline Age & 0.03 & 0.850 & & & & \\
\hline Sex & 0.10 & 0.581 & & & & \\
\hline Family history of glaucoma & 0.18 & 0.277 & & & & \\
\hline Visual acuity & 0.05 & 0.768 & & & & \\
\hline Spherical equivalent (D) & 0.09 & 0.564 & & & & \\
\hline Untreated IOP (mmHg) & 0.10 & 0.542 & & & & \\
\hline $\mathrm{CCT}(\mu \mathrm{m})$ & 0.18 & 0.281 & & & & \\
\hline DH & 0.01 & 0.973 & & & & \\
\hline Disc area $\left(\mathrm{mm}^{2}\right)$ & 0.12 & 0.474 & & & & \\
\hline ST RNFL thickness $(\mu \mathrm{m})$ & 0.22 & 0.168 & & & & \\
\hline TU RNFL thickness $(\mu \mathrm{m})$ & 0.32 & 0.048 & & & & \\
\hline TL RNFL thickness $(\mu)$ & 0.66 & $<0.001$ & 0.47 & 0.545 & $0.25,0.70$ & $<0.001$ \\
\hline IT RNFL thickness $(\mu \mathrm{m})$ & 0.50 & 0.001 & 0.16 & 0.27 & $0.01,0.31$ & 0.035 \\
\hline C2-sup $(\mathrm{dB})$ & 0.45 & 0.004 & & & & \\
\hline $\mathrm{C} 2-\inf (\mathrm{dB})$ & 0.09 & 0.597 & & & & \\
\hline Hypertension & 0.20 & 0.223 & & & & \\
\hline Diabetes mellitus & 0.11 & 0.485 & & & & \\
\hline
\end{tabular}

CCT: central corneal thickness, DH: disc hemorrhage, ST: superior-temporal, TU: temporal-upper, TL: temporal-lower, IT: inferior-temporal, RNFL: retinal nerve fiber layer, C2-sup: average total deviation (TD) values of superior 2 points within central 5 degrees, r: correlation coefficient, $\beta$ : standard partial regression coefficient, and CI: confidence interval. Values in bold are statistically significant $(P<0.2)$ in the univariate regression analysis and statistically significant $(P<0.05)$ in the multivariate regression analysis. 
TABLE 4: Univariate and multivariate regression analyses for superior mGCC thickness in the inferior visual field defect group.

\begin{tabular}{|c|c|c|c|c|c|c|}
\hline & \multicolumn{2}{|c|}{$\begin{array}{c}\text { Univariate regression } \\
\text { analysis }\end{array}$} & \multicolumn{4}{|c|}{ Multivariate regression analysis } \\
\hline & $r$ & $P$ value & Slope & $\beta$ & $95 \% \mathrm{CI}$ & $P$ value \\
\hline Age & 0.11 & 0.588 & & & & \\
\hline Sex & 0.17 & 0.400 & & & & \\
\hline Family history of glaucoma & 0.31 & 0.112 & 5.93 & 0.38 & $2.00,9.85$ & 0.005 \\
\hline Visual acuity & 0.04 & 0.836 & & & & \\
\hline Spherical equivalent (D) & 0.47 & 0.013 & & & & \\
\hline Untreated IOP (mmHg) & 0.19 & 0.354 & & & & \\
\hline CCT $(\mu \mathrm{m})$ & 0.20 & 0.317 & & & & \\
\hline $\mathrm{DH}$ & 0.19 & 0.343 & & & & \\
\hline Disc area $\left(\mathrm{mm}^{2}\right)$ & 0.40 & 0.037 & 5.15 & 0.35 & $1.43,8.87$ & 0.009 \\
\hline ST RNFL thickness $(\mu \mathrm{m})$ & 0.56 & 0.002 & & & & \\
\hline TU RNFL thickness $(\mu \mathrm{m})$ & 0.65 & $<0.001$ & 0.41 & 0.64 & $0.25,0.56$ & $<0.001$ \\
\hline TL RNFL thickness $(\mu \mathrm{m})$ & 0.01 & 0.625 & & & & \\
\hline IT RNFL thickness $(\mu \mathrm{m})$ & 0.06 & 0.767 & & & & \\
\hline C2-sup (dB) & 0.30 & 0.123 & & & & \\
\hline C2-inf (dB) & 0.24 & 0.227 & & & & \\
\hline Hypertension & 0.13 & 0.527 & & & & \\
\hline Diabetes mellitus & 0.07 & 0.718 & & & & \\
\hline
\end{tabular}

CCT: central corneal thickness, DH: disc hemorrhage, ST: superior-temporal, TU: temporal-upper, TL: temporal-lower, IT: inferior-temporal, RNFL: retinal nerve fiber layer, C2-sup: average total deviation (TD) values of superior 2 points within central 5 degrees, r: correlation coefficient, $\beta$ : standard partial regression coefficient, and CI: confidence interval. Values in bold are statistically significant $(P<0.2)$ in the univariate regression analysis and statistically significant $(P<0.05)$ in the multivariate regression analysis.

Since structural damages have been reported $[18,19]$ using image analysis tools such as OCT even in apparently normal VF, the definition of hemifield defects was strictly based on both VF results and OCT measurements. Furthermore, to eliminate the possible effect on the development of VF or OCT abnormality by glaucoma treatment, we only included the patients without glaucoma treatment history.

We found that central VF damage was more frequent and severe in the superior VF defect group than in the inferior VF defect group, and the C2-sup corresponding to the defective hemifields of the superior VF defect group was significantly lower than C2-inf corresponding to the defective hemifields of the inferior VF defect group, although the average TD of the superior and inferior hemifield defects was similar between the groups (Table 2). Moreover, the thickness of the superior mGCC in the inferior VF defect group was associated with the disc area (Table 4).

Previously, Hood et al. $[24,25]$, who investigated patients with POAG with parafoveal scotoma, suggested that since the optic disc is usually located superior from the horizontal line passing through the fovea, the axons of the retinal ganglion cells (RGCs) from the inferior part of the macula are condensed in the narrow part of the inferior quadrant of the disc. By contrast, a relatively wider part of the temporal quadrant of the disc contains axons from RGCs in the region that includes the RGCs of the superior macula and some of the RGCs of the inferior macula. In addition, the inferior area of the papillomacular bundles of axons corresponding to the superior parafoveal region of the VF is relatively small, and these bundles come into the inferior disc and pass through the inferior part of the lamina cribrosa, which has larger lamina pores compared to the temporal part. This could be the reason why parafoveal scotomas appear more frequently in the superior VF. In the present study, the superior VF defect group had a higher prevalence of "defects within 5 degrees." The average TD of the superior and inferior hemifield defects was similar in the two groups; however, the C2-sup of the superior VF defect group was significantly lower than the C2-inf of the inferior VF defect group (Table 2). We also found that the mGCC thicknesses corresponding to the defective hemifields of the superior VF defect group were significantly thinner than the superior mGCC thicknesses corresponding to the defective hemifield of the inferior VF defect group (Table 2). These findings support the findings by Hood et al. [24, 25].

To investigate factors related to the thickness of MGCC, which closely influences parafoveal scotoma, we performed multivariate regression analysis. We found that TL RNFL thickness and IT RNFL thickness were significant contributing factors to the corresponding inferior mGCC thickness in 40 eyes with the superior VF defect group (Table 3). By contrast, in 27 eyes with the inferior VF defect group, the disc area, TU RNFL thickness, and family history of glaucoma were significant contributing factors to the corresponding superior mGCC thickness (Table 4). The factors related to the reduction of the corresponding mGCC thickness may differ between the superior VF defect and inferior VF defect groups.

Our novel finding is that the optic disc area was a contributing factor to superior mGCC thickness in the inferior VF defect group (Table 4). No study has reported a correlation between the disc area and the inferior hemifield defect; however, we found a significant correlation between the disc area and superior mGCC thickness in the inferior VF defect group using multivariate regression analysis. Studies evaluating differences between patients with parafoveal and peripheral scotoma $[2,3,13-17,26]$ found no differences in the optic disc 
area between the two groups. However, in our previous study [27], we found that the thicknesses of cpRNFL and mGCC were significantly positively correlated with the disc area in eyes with glaucoma. Smaller discs may have thinner cpRNFL and mGCC and be susceptible to the inferior VF defect.

We also found that the prevalence of "defects within 5 degrees" was higher in the superior VF defect group than in the inferior VF defect group, although there was no significant difference in the prevalence of "defects within 10 degrees" between the two groups. The average TD of the superior and inferior hemifield defects in the two groups was similar (Table 2). Therefore, VF damages in the two groups were comparable. However, in the parafoveal region, the C2sup corresponding to the defective hemifields of the superior VF defect group was lower than C2-inf corresponding to the defective hemifields of the inferior VF defect group (Table 2). This suggested that visual sensitivity in the parafoveal region was further reduced in the superior VF defect group than in the inferior VF defect group. According to the studies comparing factors associated with initial parafoveal and peripheral scotomas between patients with glaucoma $[2,3,13,14,26]$, patients with initial peripheral scotoma showed inferior hemifield defects more frequently than patients with initial parafoveal scotoma. Although our study design comparing superior to inferior hemifield defects differed from the ones of previous studies, our findings supported the results of former studies $[2,3,13,14,26]$.

Mikelberg et al. [1] reported that there was no difference in the prevalence of family history of glaucoma between superior and inferior hemifield defects groups, which was also reported for initial parafoveal and peripheral scotomas between patients with glaucoma in previous studies $[25,26,28,29]$. Although the prevalence of positive family history was comparable between the two groups (Table 2), family history of glaucoma was found to be a significant contributing factor to superior mGCC thickness in the inferior VF defect group (Table 4). Further research is required to elucidate the relationship between positive family history and glaucomatous abnormality pattern on VF and OCT.

Although we excluded patients with high myopia (over -6.00) from the study, we did not find any difference in the degree of myopia between the superior and inferior VF defect groups. Similarly, findings have been reported by previous studies evaluating differences between patients with initial parafoveal and initial peripheral scotoma [2, 13-15, 26]. However, Jung et al. [3] reported that patients with initial parafoveal scotoma tended to be more myopic compared to patients with initial peripheral scotoma. Furthermore, Sung et al. [17] reported that myopic patients with NTG showed a higher prevalence of superior hemifield defects, and Park et al. [29] also reported that superior VF defects were more prevalent in myopic patients with NTG with axial length of more than $24.0 \mathrm{~mm}$. They concluded that the optic disc tilt and torsion might have influenced the location of VF defects. Moreover, refractive error and axial length are reportedly closely correlated $[26,29]$. In the current study, we could not include axial length, optic disc tilt, or torsion as factors in the multivariate regression analysis because of missing of data. Although the patients included in this study did not have high myopia, myopiainduced structural changes in the posterior pole of the eye have been reported $[30,31]$. Future studies are needed to clarify the influence of axial length, the degrees of disc tilt, and torsion angle on the pattern of VF defects [17, 26, 29].

There was no difference in age, sex, and CCT between the two groups (Table 2). This finding was in line with those of former studies [2, 3, 13-17, 26].

Previous studies have reported that patients with POAG with diabetes mellitus showed higher prevalence of inferior VF defect $[32,33]$. The lack of significant difference regarding diabetes mellitus between the two groups in this study (Table 2) may be due to the small sample size.

Previous studies have found a higher prevalence of systemic hypertension in patients with superior peripheral scotoma than in those with superior parafoveal scotoma [15]. Conversely, another study reported a higher prevalence of systemic hypotension in patients with peripheral scotoma [2]. However, most previous studies have found no difference in the prevalence of systemic hypertension between patients with parafoveal and peripheral scotoma [2, 3, $13,16,25]$. We also did not find a difference in the prevalence of systemic hypertension between the superior and inferior VF defect groups (Table 2).

Patients with POAG with high-pretreatment IOP $(>21 \mathrm{mmHg}$ ) develop parafoveal scotomas more frequently than peripheral scotomas [2], although this has not been reported yet in so-called NTG eyes [3, 13, 15-17, 25]. In the present study, 2 eyes (40\%) of 5 eyes with POAG and 24 eyes (38.7\%) of 62 eyes with NTG had "defects within 5 degrees." Although there was no significant difference in the prevalence of parafoveal scotomas between NTG and POAG $(P=0.65)$, this might be due to the small sample size.

DH is a well-known, definite risk factor for the progression of glaucoma [34-40]. Some studies have demonstrated a significantly higher rate of $\mathrm{DH}$ in patients with parafoveal scotoma than in those with peripheral scotoma $[2,15]$. However, others have not reported similar findings $[3,13,15,25]$. The present study also did not find any differences in the prevalence of $\mathrm{DH}$ between the superior and inferior VF defect groups (Table 2). This may be in part due to the differences in patient follow-up periods.

This study has some limitation including a retrospective design. We assessed the prevalence of systemic risk factors based on patient recall. Although eyes with high myopia (over $-6.00 \mathrm{D}$ ) were excluded from the study, we did not measure axial length, optic disc tilt, or torsion. Future studies are needed to clarify the influence of these factors in glaucomatous eyes with myopia.

\section{Conclusions}

We investigated the factors related to superior or inferior hemifield defects in POAG. The definitions of hemifield defects were based on both VF results and OCT measurements. We found that there was no significant difference regarding cpRNFL thickness corresponding to the superior or inferior defective hemisphere. However, the inferior mGCC thickness corresponding to the superior VF defect 
group was significantly thinner than the superior mGCC thickness corresponding to the inferior VF defect group. Although the average TD of the superior and inferior VF defects was similar between the groups, paracentral VF damage may be more frequent and severe in the superior $\mathrm{VF}$ defect group than in the inferior VF defect group. In this study, we found for the first time that the disc area was related to superior mGCC thickness in the inferior VF defect group, and this suggests that the factors related to the reduction of the corresponding mGCC thickness may differ between superior VF defect and inferior VF defect groups.

\section{Data Availability}

The data used to support the findings of this study are available from the corresponding author upon request.

\section{Conflicts of Interest}

The authors declare that there are no conflicts of interest regarding the publication of this paper.

\section{Acknowledgments}

The authors deeply thank Mr. Takashi Sato, their photographer, for data acquisition of the OCT measurements.

\section{References}

[1] F. S. Mikelberg and S. M. Drance, "The mode of progression of visual field defects in glaucoma," American Journal of Ophthalmology, vol. 98, no. 4, pp. 443-445, 1984.

[2] S. C. Park, C. G. De Moraes, C. C. W. Teng, C. Tello, J. M. Liebmann, and R. Ritch, "Initial parafoveal versus peripheral scotomas in glaucoma: risk factors and visual field characteristics field characteristics," Ophthalmology, vol. 118, no. 9, pp. 1782-1789, 2011.

[3] K. I. Jung, H.-Y. L. Park, and C. K. Park, "Characteristics of optic disc morphology in glaucoma patients with parafoveal scotoma compared to peripheral scotoma," Investigative Opthalmology \& Visual Science, vol. 53, no. 8, pp. 4813-4820, 2012.

[4] D. F. Garway-Heath, D. Poinoosawmy, F. W. Fitzke, and R. A. Hitchings, "Mapping the visual field to the optic disc in normal tension glaucoma eyes," Ophthalmology, vol. 107, no. 10, pp. 1809-1815, 2000.

[5] A. Ferreras, L. S. E. Pablo, D. F. Garway-Heath, P. Fogagnolo, and J. N. Garci'a-Feijoo, "Mapping standard automated perimetry to the peripapillary retinal nerve fiber layer in glaucoma," Investigative Opthalmology \& Visual Science, vol. 49, no. 7, pp. 3018-3025, 2008.

[6] A. Kanamori, M. Naka, A. Nagai-Kusuhara, Y. Yamada, M. Nakamura, and A. Negi, "Regional relationship between retinal nerve fiber layer thickness and corresponding visual field sensitivity in glaucomatous eyes," Archives of Ophthalmology, vol. 126, no. 11, pp. 1500-1506, 2008.

[7] F. K. Horn, C. Y. Mardin, R. Laemmer et al., "Correlation between local glaucomatous visual field defects and loss of nerve fiber layer thickness measured with polarimetry and spectral domain OCT, Investigative Ophthalmology \& Visual Science, vol. 50, no. 5, pp. 1971-1977, 2009.

[8] S. K. Gardiner, C. A. Johnson, and G. A. Cioffi, "Evaluation of the structure-function relationship in glaucoma,"
Investigative Opthalmology \& Visual Science, vol. 46, no. 10, pp. 3712-3717, 2005.

[9] R. A. Hitchings and S. A. Anderton, "A comparative study of visual field defects seen in patients with low-tension glaucoma and chronic simple glaucoma," British Journal of Ophthalmology, vol. 67, no. 12, pp. 818-821, 1983.

[10] J. Caprioli and G. L. Spaeth, "Comparison of visual field defects in the low-tension glaucomas with those in the hightension glaucomas," American Journal of Ophthalmology, vol. 97, no. 6, pp. 730-737, 1984.

[11] M. Motolko, S. M. Drance, and G. R. Douglas, "Visual field defects in low-tension glaucoma," Archives of Ophthalmology, vol. 100, no. 7, pp. 1074-1077, 1982.

[12] D. King, S. M. Drance, G. Douglas, M. Schulzer, and K. Wijsman, "Comparison of visual field defects in normaltension glaucoma and high-tension glaucoma," American Journal of Ophthalmology, vol. 101, no. 2, pp. 204-207, 1986.

[13] H.-k. Cho, J. Lee, M. Lee, and C. Kee, "Initial central scotomas vs peripheral scotomas in normal-tension glaucoma: clinical characteristics and progression rates," Eye, vol. 28, no. 3, pp. 303-311, 2014.

[14] A. Rao and S. Mukherjee, "Anatomical attributes of the optic nerve head in eyes with parafoveal scotoma in normal tension glaucoma," PLoS One, vol. 9, no. 3, Article ID e90554, 2014.

[15] J. M. Kim, H. Kyung, S. H. Shim, P. Azarbod, and J. Caprioli, "Location of initial visual field defects in glaucoma and their modes of deterioration," Investigative Opthalmology \& Visual Science, vol. 56, no. 13, pp. 7956-7962, 2015.

[16] J.-W. Kang, B. Park, and B. J. Cho, "Comparison of risk factors for initial central scotoma versus initial peripheral scotoma in normal-tension glaucoma," Korean Journal of Ophthalmology, vol. 29, no. 2, pp. 102-108, 2015.

[17] M. S. Sung, H. Heo, Y. S. Ji, and S. W. Park, "Predicting the risk of parafoveal scotoma in myopic normal tension glaucoma: role of optic disc tilt and rotation," Eye, vol. 31, no. 7, pp. 1051-1059, 2017.

[18] S. T. Takagi, Y. Kita, F. Yagi, and G. Tomita, "Macular retinal ganglion cell complex damage in the apparently normal visual field of glaucomatous eyes with hemifield defects," Journal of Glaucoma, vol. 21, no. 5, pp. 318-325, 2012.

[19] D. S. Grewal, M. Sehi, and D. S. Greenfield, "Diffuse glaucomatous structural and functional damage in the hemifield without significant pattern loss," Archives of Ophthalmology, vol. 127, no. 11, pp. 1142-1148, 2009.

[20] A. Iwase, Y. Suzuki, M. Araie et al., "The prevalence of primary open-angle glaucoma in Japanese," Ophthalmology, vol. 111, no. 9, pp. 1641-1648, 2004.

[21] D. R. Anderson and V. M. Patella, Automated Static Perimetry, Mosby, Maryland Heights, MI, USA, 2nd edition, 1990.

[22] A. Anraku, K. Ishida, and N. Enomoto, "Association between optic nerve head microcirculation and macular ganglion cell complex thickness in eyes with untreated normal tension glaucoma and a hemifield defect," Journal of Ophthalmology, vol. 2017, Article ID 3608396, 9 pages, 2017.

[23] S. Nakagawa, H. Murata, H. Saito et al., "Frequency doubling technology for earlier detection of functional damage in standard automated perimetry-normal hemifield in glaucoma with low-to-normal pressure," Journal of Glaucoma, vol. 21, no. 1, pp. 22-26, 2012.

[24] D. C. Hood, A. S. Raza, C. G. V. de Moraes et al., "Initial arcuate defects within the central 10 degrees in glaucoma," Investigative Opthalmology \& Visual Science, vol. 52, no. 2, pp. 940-946, 2011. 
[25] D. C. Hood, A. S. Raza, C. G. V. de Moraes, J. M. Liebmann, and R. Ritch, "Glaucomatous damage of the macula," Progress in Retinal and Eye Research, vol. 32, pp. 1-21, 2013.

[26] J. A. Choi, H.-Y. L. Park, and C. K. Park, "Difference in the posterior pole profiles associated with the initial location of visual field defect in early-stage normal tension glaucoma," Acta Ophthalmologica, vol. 93, no. 2, pp. e94-e99, 2015.

[27] N. Enomoto, A. Anraku, K. Ishida, A. Takeyama, F. Yagi, and G. Tomita, "Size of the optic nerve head and its relationship with the thickness of the macular ganglion cell complex and peripapillary retinal nerve fiber layer in patients with primary open angle glaucoma," Journal of Ophthalmology, vol. 2015, Article ID 186249, 6 pages, 2015.

[28] D. C. Hood, A. Slobodnick, A. S. Raza, C. G. de Moraes, C. C. Teng, and R. Ritch, "Early glaucoma involves both deep local, and shallow widespread, retinal nerve fiber damage of the macular region," Investigative Opthalmology \& Visual Science, vol. 55, no. 2, pp. 632-649, 2014.

[29] H.-Y. L. Park, K. Lee, and C. K. Park, "Optic disc torsion direction predicts the location of glaucomatous damage in normal-tension glaucoma patients with myopia," Ophthalmology, vol. 119, no. 9, pp. 1844-1851, 2012.

[30] H. A. Quigley, E. M. Addicks, W. R. Green, and A. E. Maumenee, "Optic nerve damage in human glaucoma," Archives of Ophthalmology, vol. 99, no. 4, pp. 635-649, 1981.

[31] C. Mayama, Y. Suzuki, M. Araie et al., "Myopia and advancedstage open-angle glaucoma," Ophthalmology, vol. 109, no. 11, pp. 2072-2077, 2002.

[32] J. H. Zeiter, D. H. Shin, and N. H. Baek, "Visual field defects in diabetic patients with primary open-angle glaucoma," American Journal of Ophthalmology, vol. 111, no. 5, pp. 581-584, 1991.

[33] J. H. Zeiter and D. H. Shin, "Diabetes in primary open-angle glaucoma patients with inferior visual field defects," Graefe's Archive for Clinical and Experimental Ophthalmology, vol. 232, no. 4, pp. 205-210, 1994.

[34] P. R. Healey, P. Mitchell, W. Smith, and J. J. Wang, "Optic disc hemorrhages in a population with and without signs of glaucoma," Ophthalmology, vol. 105, no. 2, pp. 216-223, 1998.

[35] D. L. Budenz, D. R. Anderson, W. J. Feuer et al., "Detection and prognostic significance of optic disc hemorrhages during the ocular hypertension treatment study," Ophthalmology, vol. 113, no. 12, pp. 2137-2143, 2006.

[36] M. C. Leske, A. Heijl, L. Hyman et al., "Predictors of longterm progression in the early manifest glaucoma trial," Ophthalmology, vol. 114, no. 11, pp. 1965-1972, 2007.

[37] S. Drance, D. R. Anderson, and M. Schulzer, "Risk factors for progression of visual field abnormalities in normal-tension glaucoma," American Journal of Ophthalmology, vol. 131, no. 6, pp. 699-708, 2001.

[38] S. W. Siegner and P. A. Netland, "Optic disc hemorrhages and progression of glaucoma,” Ophthalmology, vol. 103, no. 7, pp. 1014-1024, 1996.

[39] S. Komori, K. Ishida, and T. Yamamoto, "Results of long-term monitoring of normal-tension glaucoma patients receiving medical therapy: results of an 18-year follow-up," Graefe's Archive for Clinical and Experimental Ophthalmology, vol. 252, no. 12, pp. 1963-1970, 2014.

[40] K. Ishida, T. Yamamoto, K. Sugiyama, and Y. Kitazawa, "Disk hemorrhage is a significantly negative prognostic factor in normal-tension glaucoma," American Journal of Ophthalmology, vol. 129, no. 6, pp. 707-714, 2000. 


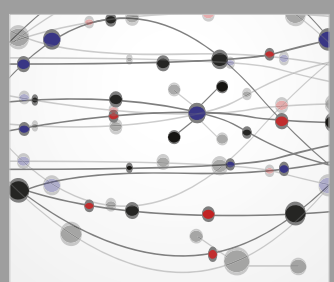

The Scientific World Journal
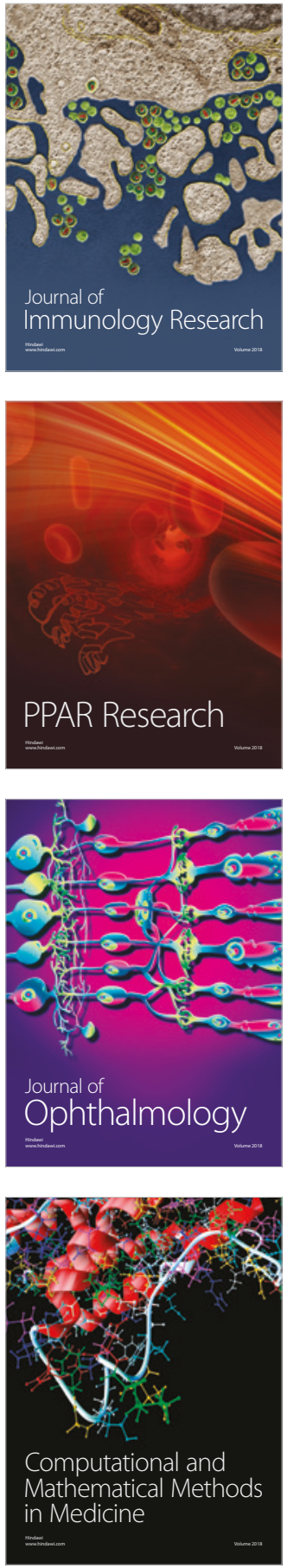

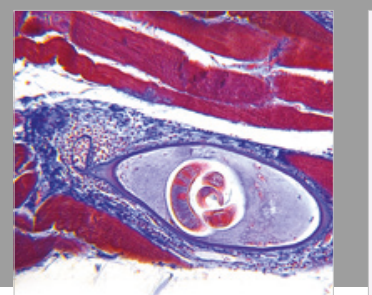

Gastroenterology Research and Practice

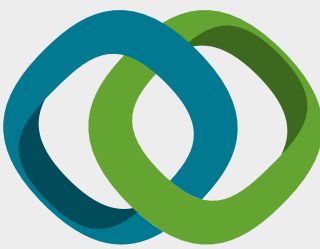

\section{Hindawi}

Submit your manuscripts at

www.hindawi.com
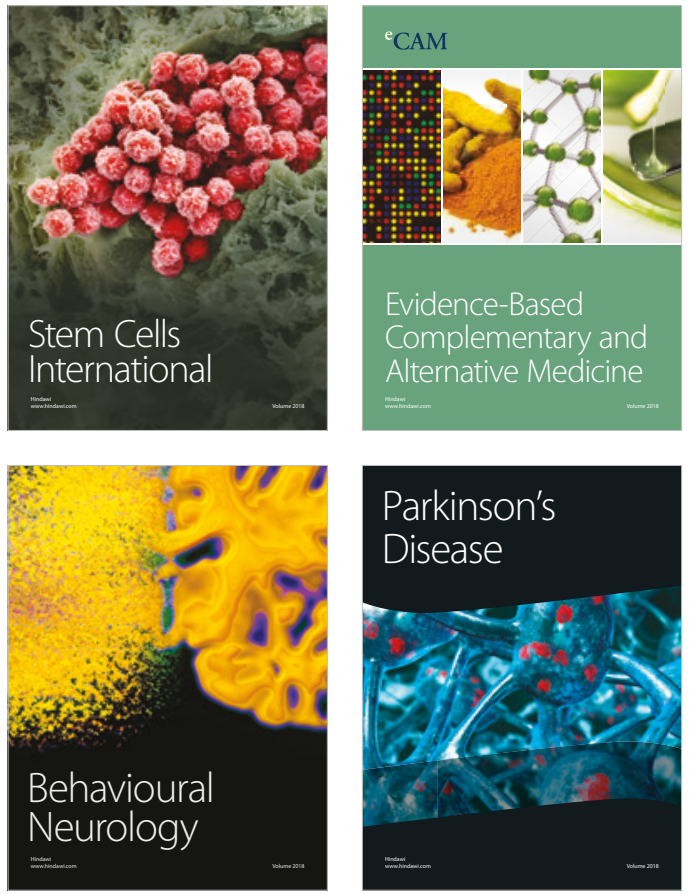

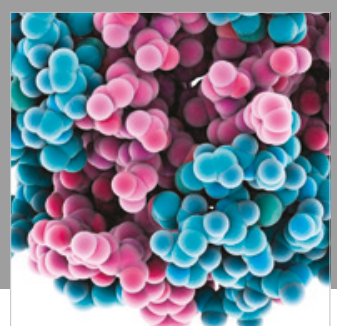

ournal of

Diabetes Research

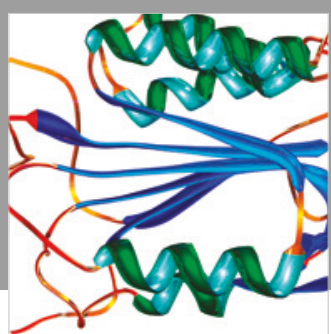

Disease Markers
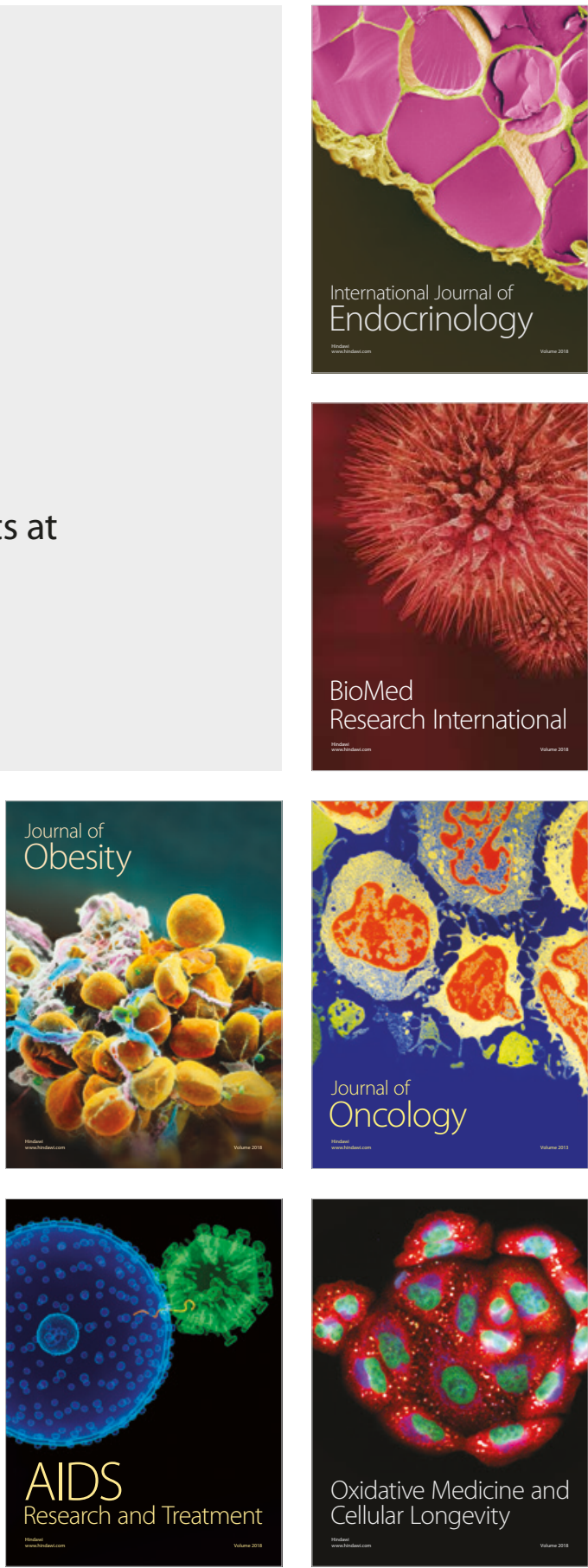